\title{
O Cloze como instrumento de avaliação de leitura nas séries iniciais
}

\author{
Márcia Maria Peruzzi Elia da Mota \\ Universidade Salgado de Oliveira / Universidade do Estado do Rio de Janeiro - RJ \\ Acácia Aparecida Angeli dos Santos \\ Universidade São Francisco - SP
}

\section{Resumo}

A literatura na área tem apontado a importância das habilidades referentes à leitura e escrita, especialmente quando se estuda o processo de alfabetização e suas dificuldades. Buscando verificar a validade do Cloze como instrumento de avaliação de leitura, o presente estudo foi elaborado tendo como principal objetivo comparar os escores do Cloze com o Teste de Desempenho Escolar (TDE), que apresenta, além de estudos de validade e precisão, também uma proposta de normatização. A amostra do estudo constituiu-se de quarenta e duas crianças, das quais dezenove eram alunas da $1^{\mathrm{a}}$ série e vinte e três da $2^{\mathrm{a}}$ série (no sistema atual, $2^{\circ}$ ano e $3^{\circ}$ ano) do Ensino Fundamental de escolas particulares situadas no Interior de Minas. Os resultados mostram evidências de validade de critério e convergência para o Cloze e indicam sua utilidade como um teste de avaliação de leitura.

Palavras-chave: Teste de Cloze, alfabetização, psicometria.

\section{The Cloze Evaluation as a measure of reading ability in Primary Education}

\begin{abstract}
Literature review in this field of investigation has pointed to the importance of reading and spelling skills, especially when considering the literacy process and difficulties related to it. In order to verify the validity of the Cloze Test as reading assessment instrument, the goal of this study was to compare Cloze Test with The School Achievement Test (TDE-), which, in addition to studies of validity and accuracy, has a proposal for grade regulations. The study consisted of 42 children, 19 children from 1 st grade and 23 from 2nd grade (in the current system 2nd year and 3 rd year), from private primary education schools located in Minas Gerais - Brazil. The results reveal the validity of Cloze criterion and point to its usefulness as assessment of reading task.
\end{abstract}

Keywords: Cloze testing, literacy, psychometrics.

\section{El Cloze como instrumento de evaluación de lectura en los primeros grados escolares}

\section{Resumen}

La literatura en el área ha señalado para la importancia de las habilidades relacionadas a la lectura y escritura, especialmente cuando se estudia el proceso de alfabetización y sus dificultades. El presente estudio se elaboró teniendo como principal objetivo comparar los escores del Cloze con el Test de Rendimiento Escolar que presenta, además de estudios de validez y precisión, también una propuesta de estandarización, tratando así de verificar la validez del Cloze como instrumento de evaluación de lectura. La muestra del estudio fue de 42 niños, siendo 19 niñas alumnas del $1^{\text {er }}$ grado y 23 del $2^{\circ}$ grado (en el sistema actual $2^{\circ}$ y $3^{\circ}$ años), ambos de la enseñanza fundamental de escuelas particulares, situadas en el interior del Estado de Minas Gerais. Los resultados muestran evidencias de validez de criterio y convergente para el Cloze y indican su utilidad como un test de evaluación de lectura.

Palabras Clave: Test de Cloze, alfabetización, psicometría. 


\section{Introdução}

A técnica de Cloze está entre os primeiros procedimentos sistemáticos utilizados na avaliação da compreensão em leitura, compreensão que significa a competência de dar o sentido ao texto, valendo-se de dois principais tipos de processamento: a redundância semântica e sintática do texto e seus conhecimentos prévios. Destaca-se que o uso do Cloze é compatível com a concepção do processo de compreensão em leitura baseada nos modelos de integração recentemente propostos, que atribuem importância tanto ao conhecimento linguístico como ao conceitual (Kintsch, 1978, 1994). Além das vantagens já assinaladas, o Cloze é mais eficiente que os testes em formato de questões sobre o entendimento do texto, pois um escore alto em um teste desse tipo não significa necessariamente que o estudante tenha compreendido bem o texto, mas apenas que possui uma boa técnica para responder corretamente às questões.

Há evidências de validade estabelecidas em estudos brasileiros que apontam o Cloze como uma medida de inteligibilidade do texto (Melo, 1986; Molina, 1979), bem como uma medida de compreensão de leitura (Cunha \& Santos, 2006, 2009, 2010; Santos, Primi, Taxa, \& Vandramini, 2002). Outro aspecto importante a ser observado é que, mesmo sendo um teste de construção e correção bastante simples, como já referido, sua utilização deve ser criteriosa, para que sejam evitados problemas graves em sua interpretação e, consequentemente, haja comprometimento no resultado obtido caso no qual ele seria inválido enquanto recurso diagnóstico, com as consequentes implicações educacionais (Rupp, Ferne, \& Choi, 2006).

Quanto à preparação de um texto para ser estruturado seguindo a técnica de Cloze, várias são as diversificações possíveis em relação à forma original proposta por Taylor (1953). Sobre esse aspecto, Abraham e Chapelle (1992) consideram que a estruturação do texto deve ser de acordo com dois níveis de dificuldade. O primeiro é classificado como micronível, e nele são utilizados itens relativamente fáceis de compreender, enquanto o chamado macronível exige grande abstração e apresenta um alto grau de dificuldade. Por sua vez, os determinantes da dificuldade implicada em um item podem ser divididos de acordo com os fatores intrínsecos e extrínsecos. Os fatores intrínsecos dependem de alguns elementos estruturais do texto, que facilitam a execução da tarefa e funcionam como um estímulo para o entendimento do item; já os fatores extrínsecos incluem os conhecimentos que o estudante tem sobre o assunto e as estratégias utilizadas para o desempenho da tarefa.

Corroborando os resultados e as conclusões obtidas por Abraham e Chapelle (1992), a pesquisa de Santos e cols. (2002), valendo-se da análise da Teoria de Resposta ao Item, indicou que os acertos no Cloze não dependem somente da habilidade dos sujeitos, mas também das dificuldades dos itens que compõem o texto. Ressalta-se que, com base no estudo dos itens do Cloze, é possível abrir mais um leque de investigações científicas com resultados promissores, que permitirão a ampliação do conhecimento sobre esse instrumento.

A correção do Cloze é bastante simples, como mencionado, podendo basear-se em dois critérios. O primeiro é denominado correção verbatim ou literal, e nele se aceita como correta unicamente a palavra idêntica à que foi excluída do texto. O segundo critério, pelo qual se aceita como correta uma palavra de sentido equivalente ou outra palavra adequada ao contexto, é conhecido como correção sinônima. Para efeitos de diagnóstico e de pesquisa é recomendada a primeira forma, que evita a subjetividade e facilita a correção (Bitar, 1989; Chance, 1985; McKenna, 1979).

Pesquisas estrangeiras têm apontado o teste de Cloze como um instrumento válido para avaliar a compreensão em leitura em qualquer etapa de escolarização (do Ensino Fundamental ao Superior), podendo ser utilizado como preditivo do desempenho acadêmico (Barnitz, 1998; Riley, 1986). No Brasil, muitos dos estudos utilizando esse instrumento têm sido realizados com universitários, alguns deles para avaliação da compreensão em leitura, e outros, com o propósito de remediação, são empregados com estudantes que apresentam déficits de leitura (Oliveira \& Santos, 2008; Sampaio \& Santos, 2002; Silva \& Santos, 2004, entre outros). Estudos com crianças e adolescentes também vêm sendo realizados, especialmente em programas de desenvolvimento da compreensão em leitura (Mota e cols., 2009; Santos, 2005; Santos \& Oliveira, 2010; Zucoloto \& Sisto, 2002).

Verifica-se em vários dos estudos referidos que os textos infantis estruturados no padrão da técnica de Cloze são elaborados com menor número de palavras e, consequentemente, de omissões. Ao lado disso, tais textos têm sido utilizados como parâmetro da eficácia da intervenção realizada ou como procedimento específico para o desenvolvimento da compreensão com o emprego de diferentes variações que a técnica possibilita. Não obstante, são escassos os estudos especificamente desenvolvidos para identificar as evidências de validade do teste no Brasil. Assim, pretende-se nesta investigação focalizar o Teste de Cloze para avaliação da compreensão de leitura em crianças das séries iniciais do Ensino Fundamental, sendo suas características detalhadamente descritas no método.

Outros estudos têm apresentado evidências de validade para os testes de Cloze aqui utilizados, fornecendo elementos que apoiam a pertinência dos textos como medidas da compreensão de leitura. A pesquisa de Cunha e Santos (2009) objetivou encontrar evidências de validade por processo de resposta para o teste de Cloze, explorando as diferenças qualitativas nos erros cometidos por crianças durante o preenchimento dos espaços de um teste de Cloze. De um banco de dados com mais de 200 protocolos, foram escolhidos alguns que formaram dois grupos extremos: alunos com médias baixas $(n=22)$ e alunos com médias altas $(n=22)$. As autoras consideraram que os resultados encontrados evidenciavam diferenças entre bons leitores (crianças com médias altas), que cometeram mais erros lexicais, enquanto os maus leitores (crianças com médias baixas) cometeram mais erros semânticos, haja vista que os 
erros semânticos são indicadores de falta de compreensão de leitura, especialmente ao serem comparados com erros lexicais decorrentes apenas da substituição da palavra esperada por outra palavra sinônima.

Em trabalho subsequente, Cunha e Santos (2010) analisaram o desempenho de 266 crianças de idade entre oito e treze anos, de terceiras e quartas séries do Ensino Fundamental de escolas públicas, particulares e do SESI em crianças paulistas. O objetivo foi estabelecer evidências de validade convergente entre a Escala de Avaliação de Dificuldades na Aprendizagem da Escrita e o Teste de Cloze. A análise da correlação entre as médias obtidas no Cloze pelas categorias do Escala de Avaliação de Dificuldades na Aprendizagem da Escrita indicou índices de magnitude moderada, o que permitiu às autoras afirmar terem identificado evidência de validade convergente entre os instrumentos.

$O$ teste de desempenho escolar (TDE) tem sido amplamente usado para a avaliação do desempenho escolar em pesquisas brasileiras (Dias, Enumo \& Turini, 2006; Lucio, Pinheiro, \& Nascimento, 2009; Milani \& Loureiro, 2009; Siqueira \& DellÁglio, 2010). Por ser o único teste de avaliação do desempenho em leitura e escrita padronizado no Brasil, ele foi escolhido para servir como teste de referência para a comparação com o Cloze no presente estudo. Especificamente o subteste de leitura implica a avaliação da leitura em voz alta de uma lista de palavras isoladas de uma lista de palavras que é apresentada às crianças.

Os estudos aqui recuperados mostram a importância das habilidades referentes à leitura, especialmente quando se estuda o processo de alfabetização e suas dificuldades (problema hoje de grande vulto na realidade do ensino público brasileiro) para verificar evidências de validade para o Cloze enquanto instrumento de avaliação da compreensão de leitura. Assim, o presente estudo foi elaborado tendo como objetivo identificar evidências de validade relacionadas a variáveis externas, tanto a de critério, pela comparação dos resultados das séries, como também evidência de validade por comparação entre medidas de construtos relacionados, ou seja, da compreensão de leitura (Cloze) e do desempenho escolar (TDE).

\section{Método}

\section{Participantes}

A amostra do estudo constituiu-se de uma subamostra de quarenta e duas crianças que participavam de um estudo longitudinal sobre o papel das habilidades metalinguísticas e a alfabetização, das quais dezenove eramalunas da $1^{\text {a }}$ série e vinte e três da $2^{\mathrm{a}}$ série (no sistema atual, $2^{\circ}$ ano e $3^{\circ}$ ano) do Ensino Fundamental de escolas particulares situadas no Interior de Minas. A média de idade das crianças de $1^{\text {a }}$ série foi de 87,5 meses $(d p=3,93)$, e a da $2^{\mathrm{a}}$ série,de 98,3 meses $(d p=4,58)$.

\section{Instrumentos}

O Cloze, tal como criado por Taylor em 1953, consiste de um texto do qual se suprimem alguns vocábulos, pedindo-se ao leitor que preencha os espaços com as palavras que melhor completarem o sentido do texto. Para o presente estudo foram escolhidos dois textos, com histórias infantis "A princesa e o fantasma" (Cloze 1) e "Uma vingança infeliz" (Cloze 2), especialmente elaborados para serem usados nas séries iniciais do Ensino Fundamental, cujas propriedades psicométricas já tinham sido estabelecidas em estudo anterior no Brasil (Santos, 2005; Santos \& Oliveira, 2010). Assim sendo, já havia sido identificado que ambos apresentavam evidências de validade (convergente, de critério e experimental) e também índices satisfatórios de consistência interna $(\alpha>0,70)$. O texto foi preparado conforme recomendado para Clozes de razão fixa, com a eliminação dos quintos vocábulos, que foram substituídos por um traço proporcional ao tamanho da palavra eliminada. O número de acertos possíveis para cada um dos textos é de 15 pontos (correspondente ao acerto de todos os espaços preenchidos corretamente), perfazendo o total de 30 pontos quando se considera o conjunto das duas histórias.

O Teste de Desempenho Escolar (TDE) foi elaborado por Stein em 1994 e tem se mostrado um teste adequado para avaliação de alunos de 7 a 12 anos, visto que seus estudos de validade e precisão e de normatização foram feitos com sujeitos dessa faixa etária. A autora apresenta evidências de validade de conteúdo e estudos de consistência interna para o subteste de escrita, de aritmética e de leitura. O instrumento tem ainda evidências de validade de critério, uma vez que por meio dele foi possível a discriminação das séries. No presente estudo foram aplicados apenas os subtestes de leitura.

\section{Procedimento}

Após a aprovação do projeto de pesquisa pelo Comitê de Ética e da autorização das escolas, os alunos foram convidadas a participar por meio de uma carta-convite, tendo sido aplicados os instrumentos somente àqueles cujos responsáveis tivessem assinado o Termo de Consentimento Livre e Esclarecido.

As crianças foram avaliadas individualmente em três sessões, com duração total de 30 minutos. Na primeira sessão foram aplicadas várias tarefas de Consciência metalinguística; e na segunda sessão foram aplicados os dois textos de Cloze, sendo pedido à criança que respondesse um de cada vez. Os textos foram sempre apresentados na mesma ordem. Solicitava-se que lessem a história até o fim e depois voltassem ao início e preenchessem os espaços com as palavras que julgassem que dariam mais sentido ao texto. Em outra sessão foram aplicados os dois subtestes do Teste de Desempenho Escolar, também seguindo-se o procedimento de aplicação do manual. 


\section{Análise dos dados}

Para estabelecer a validade de critério do teste de Cloze, dois Testes-t não paramétricos Mann-Whitney foram utilizados, uma vez que o tamanho das amostras torna mais adequado utilizar esse tipo de teste. Para estabelecer a validade entre constructos relacionados foi possível a análise de correlação paramétrica, que foi feita entre os escores do TDE e do CLOZE. Três Testes-t não paramétricos Mann-Whitney foram usados para comparações da performance no Cloze para as três categorias observadas: superior, médio superior e médio.

\section{Resultados}

A apresentação dos resultados segue a ordem dos objetivos estabelecidos. Dessa forma, comentamos a seguir os dados obtidos em relação ao primeiro objetivo, que consistiu na comparação dos escores médios das duas séries estudadas e a comparação entre as diferentes categorias em que os alunos foram agrupados pelo TDE.

\section{Comparações entre séries}

Para verificar se o Cloze era capaz de discriminar as séries, dois Testes-t não paramétricos Mann-Whitney foram usados para comparações da performance no Cloze 1 e Cloze 2 para as duas séries estudadas. A performance das crianças no Cloze diferiu de forma estatisticamente significativa na comparações entre a primeira e segunda série nos dois subtestes $(U=-2,98 ; p=0,003$ para o Cloze 1 e $U=-3,86$; $p<0,001$ para o Cloze 2). O Cloze discriminou as séries também para o escore total, obtido pela soma dos dois subtestes $(U=-3,54 ; p=0,001)$.

\section{Comparações entre bons e maus leitores}

O TDE apresenta também uma classificação das crianças por categorias de acordo com o nível de leitura. Para a primeira série, quatro categorias de leitores são apresentadas de acordo com a pontuação do teste (superior, médio superior, médio, inferior), e para segunda série são descritas cinco categorias de leitores (superior, médio superior, médio, médio inferior, inferior). É possível explorarmos a capacidade do Cloze em discriminar as crianças por nível de leitura, comparando sua nota no Cloze em cada categoria; mas essa análise teve que ser feita para a primeira e a segunda série separadamente, pois as análises por categoria são diferentes para cada série. Os resultados obtidos estão apresentados na Tabela 1, que apresenta a média de acerto e o desvio padrão nos testes de Cloze para cada categoria.

Podemos observar que quanto melhor o desempenho no TDE, melhor o desempenho no teste de Cloze para as duas séries e categorias. Essas diferenças foram checadas por meio de análises estatísticas. Cabe ressaltar que em algumas categorias não havia crianças, e que em outras o número de crianças foi bem reduzido, por isso para essa parte das análises foram utilizados testes não paramétricos. Por exemplo, para a primeira série não havia crianças na categoria inferior, e para a segunda série as crianças foram categorizadas em três grupos: superior, médio e inferior.

Para a primeira série, como havia categorias vazias, recorremos ao teste não paramétrico Mann-Whitney para as comparações da performance no Cloze para as três categorias observadas: superior, médio superior e médio. A

Tabela 1. Média de Acerto no Cloze por Categoria de Leitura no TDE.

\begin{tabular}{|c|c|c|c|c|c|}
\hline Categoria & $\mathrm{N}$ & Série & $\begin{array}{l}\text { Cloze } 1 \text { e Desvio } \\
\text { padrão }\end{array}$ & $\begin{array}{l}\text { Cloze } 2 \text { e Desvio } \\
\text { padrão }\end{array}$ & $\begin{array}{l}\text { Média Total e } \\
\text { Desvio padrão }\end{array}$ \\
\hline Superior & 8 & Primeira série & $\begin{array}{c}7,00 \\
(2,45)\end{array}$ & $\begin{array}{c}4,12 \\
(1,88)\end{array}$ & $\begin{array}{l}11,12 \\
(4,19)\end{array}$ \\
\hline Médio Superior & 8 & & $\begin{array}{c}4,50 \\
(3,34)\end{array}$ & $\begin{array}{c}2,25 \\
(2,12)\end{array}$ & $\begin{array}{c}6,75 \\
(4,89)\end{array}$ \\
\hline Médio & 3 & & $\begin{array}{l}2,33 \\
(1,5)\end{array}$ & $\begin{array}{c}0,67 \\
(0,57)\end{array}$ & $\begin{array}{c}3,00 \\
(1,00)\end{array}$ \\
\hline Superior & 7 & Segunda série & $\begin{array}{c}9,7 \\
(2,69)\end{array}$ & $\begin{array}{c}6,57 \\
(1,51)\end{array}$ & $\begin{array}{l}16,28 \\
(3,82)\end{array}$ \\
\hline Médio & 7 & & $\begin{array}{c}8,38 \\
(2,33)\end{array}$ & $\begin{array}{c}5,87 \\
(1,96)\end{array}$ & $\begin{array}{l}14,15 \\
(3,60)\end{array}$ \\
\hline Inferior & 3 & & $\begin{array}{c}5,7 \\
(3,2)\end{array}$ & $\begin{array}{c}4,67 \\
(3,21)\end{array}$ & $\begin{array}{l}10,33 \\
(6,35)\end{array}$ \\
\hline
\end{tabular}


performance das crianças no Cloze diferiu de forma estatisticamente significativa na comparação entre o nível superior e médio $(U=1,0 ; p=0,02$ para o Cloze 1 e $U=1,0 ; p=0,02$ para o Cloze 2). Na comparações entre os grupos superior e médio superior $(U=17,5 ; p=0,12$ para o Cloze 1 e $U=14,5 ; p=0,06$ para o Cloze 2) e nas comparações entre médio superior e médio, os resultados não foram estatisticamente significativos ( $U=7,5 ; p=0,35$ e $U=7,0 ; p=0,29$ respectivamente).

Comparações no escore total do Cloze também mostraram diferenças entre os grupos. Na comparação entre os grupos médio e superior, $U=-2,36, p=0,018$. Para os grupos médio superior e médio não houve diferenças significativas $(U=0,21, p=0,27)$, e para o superior e o médio superior os resultados também não foram significativos $(U=0,1 ; p=0,1)$.

O mesmo tipo de análise foi feito para a segunda série. Na segunda-série não houve diferenças estatísticas para comparações em nenhum dos grupos. Os resultados da análise de variância não paramétrica mostraram que as comparações entre os grupos não produziram diferenças significativas $(U=3,9 ; p=0,14$ para o Cloze 1 e $U=1,57$; $p=0,45$ para o Cloze 2 e para o escore total $U=2,5 ; p=0,28)$, o que pode ser atribuído ao acaso.

Para alcançar o segundo objetivo proposto, foi analisada a correlação entre as medidas usadas, pressupondo-se que as medidas (Cloze e TDE) avaliam construtos relacionados. Importante lembrar que o Cloze avalia a compreensão de leitura e os dois subtestes do TDE avaliam o desempenho escolar especificamente no que se refere à leitura e à escrita.

\section{Correlações entre o Cloze e o TDE}

Um escore global do teste de Cloze foi computado, somando-se o número de acerto nos dois testes usados (Cloze 1 + Cloze 2). O escore final do Cloze apresentou uma distribuição normal, por isso foram realizadas análises paramétricas nos dados. Para investigar se o Cloze e o TDE avaliam habilidades semelhantes, recorreu-se à prova de correlação de Pearson. A variável selecionada para análise no TDE foi o escore bruto das crianças no teste de leitura. O resultado da análise mostra correlações positivas e significativas moderadas entre a soma dos escores dos dois testes de Cloze e os escores no TDE $(r=0,47 ; p<0,001)$.

Para explorar as correlações entre as séries específicas foram feitas as correlações não paramétricas de Spearman. Os resultados corroboram os encontrados para o escore global. Para o segundo ano a correlação entre o TDE e o Cloze mostrou índices de correlação moderados e significativos $(r=0,48$ e $p=0,034)$, e resultados semelhantes foram encontrados para o terceiro ano $(r=0,41$ e $p=0,048)$.

\section{Discussão}

Ressaltamos na introdução desse estudo que literatura na área tem apontado a importância das habilidades de leitura para o sucesso escolar, especialmente quando se estuda o processo de alfabetização e suas dificuldades, problema de recorrente discussão na realidade do ensino público brasileiro. Buscar instrumentos de avaliação de fácil aplicação e com boas qualidades psicométricas é importante no processo de identificar as dificuldades de leitura, para posteriormente realizar programas de remediação, especialmente porque algumas práticas educativas podem prevenir a ocorrência dos chamados problemas de aprendizagem (Santos, 2004; Santos \& Oliveira, 2010; Zucoloto \& Sisto, 2002).

Obtivemos evidências de validade de critério pela comparação entre séries. As crianças da primeira série tiveram desempenho inferior aos das de segunda-série para o escore total no Cloze e nas análises individuais de cada subteste. Esses resultados mostram que o Cloze é uma boa alternativa para avaliar o desempenho das crianças em leitura nessa faixa etária, uma vez que discrimina o desempenho dos alunos de diferentes níveis de habilidades. Os dados aqui obtidos são congruentes com os de outros estudos que focalizam amostras de alunos de séries iniciais do Ensino Fundamental. Há congruência entre os resultados obtidos, especialmente se considerarmos aqueles obtidos por Cunha e Santos em 2010.

Para a análise, que tinha como propósito a discriminação entre grupos de leitores baseada no desempenho na leitura, os resultados foram menos claros. Dividimos as crianças nas categorias de desempenho da leitura sugeridas pelo TDE. Para cada categoria observamos a média de acerto nos dois testes de Cloze. Esperava-se que as médias no Cloze fossem compatíveis com a classificação baseada nos níveis de leitura no TDE, o que de fato aconteceu para o padrão geral das médias. As crianças com melhor desempenho no TDE tiveram melhores escores no teste de Cloze para cada grupo de leitores estudados.

Essas diferenças foram testadas estatisticamente; mas quando essas análises foram efetuadas os resultados não foram tão claros. As crianças de primeira série classificadas no nível superior de leitura no TDE tiveram performance superior à do grupo médio para os dois testes de Cloze. Esses resultados eram os esperados se o teste de Cloze estivesse discriminando bons e maus leitores; mas para as comparações entre o grupo superior e médio superior e para comparações entre médio superior e médio os resultados não foram estatisticamente significativos. Esse resultado pode dever-se ao fato de as diferenças entre os grupos médio, médio superior e superior poderem ser sutis demais para serem percebidas pelo teste de Cloze. Entende-se que esse resultado não compromete a utilização do teste para discriminar bons e maus leitores. Este resultado revela uma limitação do presente estudo, visto que pode ser atribuído a características idiossincráticas da amostra, devendo ser aplicado em amostras maiores e mais diversificadas, incluindo também crianças da rede pública.

Por outro lado, o mesmo tipo de análise foi feito para a segunda série, e nesse caso não houve diferenças estatísticas para comparações em nenhum dos grupos. Os 
resultados nesse caso não puderam ser explicados por efeito de teto. Houve variabilidade nos escores. Uma possível explicação para esses resultados pode estar relacionada a uma limitação do presente estudo no que diz respeito ao tamanho da amostra. Havia apenas três crianças no grupo de leitores de nível inferior. A análise do desvio padrão desse grupo também mostra grande variabilidade $(d p=6,3)$. É possível que, se este estudo fosse realizado com um grupo maior de crianças, os resultados tivessem sido estatisticamente significativos, uma vez que as médias de acerto observadas seguem os padrões esperados pelos níveis de leitura do TDE, isto é, os alunos do grupo inferior tiveram os menores escores, e os escores melhoram gradativamente para os grupos médio e superior.

Em suma, embora os resultados não tenham sido significativos para todas as séries, os padrões das médias no Cloze condizem com a classificação do TDE. As crianças com escores inferiores tiveram médias mais baixas que as crianças com escores médios e superiores. Apesar de serem necessárias novas análises com amostras de crianças nas séries iniciais, de um modo geral o teste de Cloze aqui apresentado parece ser uma medida adequada para avaliar a leitura de crianças nos estágios iniciais de aprendizado da escrita.

As análises subsequentes feitas nos dados corroboram esses resultados. Buscamos verificar também a validade do Cloze como instrumento de avaliação de leitura correlacionando os escores do Cloze com o Teste de Desempenho Escolar (TDE), que apresenta, além de estudos de validade e precisão, também uma proposta de normatização.Nossos resultados mostram correlações significativas e positivas entre e Cloze e o subteste de leitura do TDE, indicando que esses dois testes envolvem habilidades semelhantes e atestam a validade do Cloze como teste de avaliação de leitura.

O fato de essa correlação entre as variáveis ser moderada e não forte deve-se possivelmente a diferenças entre o processamento da leitura contextual(como no Cloze) e de palavras isoladas (como no TDE). A leitura de palavras isoladas pode ser feita por uma rota visual, sem conversão fonológica, por acesso direto à pronúncia ou significado. Neste caso essa identificação pode ocorrer pela análise da forma da palavra ou pela identificação das sequências de letras (Ellis, 1995); ou poderia ser feita pela rota alfabética, pela conversão letra e som. A leitura de palavras no contexto pode envolver esses dois tipos de reconhecimento, sendo que ambos os tipos de processamento ainda podem estar associados ao uso de pistas sintático-semânticas.

Um último ponto a ressaltar é a importância prática desses achados. Dada a importância que a leitura tem na vida do estudante, verificamos um grande número de estudos que investigam o processo de aquisição da leitura no início dos anos escolares (Brandão \& Spinillo, 1998; Mota e cols., 2009; Nicholson, 1993; Rego, 1995; Spinillo \& Mahon, 2007; Tunmer, 1990). À medida que avançam os anos de escolarização, o número de estudos se reduz substancialmente, especialmente no tocante ao período da adolescência. Cantrell, Almasi, Carter, Rintamaa e Madden
(2010) chamam a atenção para o fato de que os problemas de compreensão de texto muitas vezes persistem durante a adolescência e de que há poucos estudos sobre o entendimento da compreensão leitora nessa faixa etária. No Brasil, estudos de Oliveira, Boruchovitch e Santos (2008) e Oliveira e Santos (2008) produziram resultados a partir do Cloze para suprir essa lacuna na área.

Entender o processo de aquisição da leitura durante todo o período escolar é de grande importância. Pelos estudos recuperados na literatura sabe-se que o Cloze é uma alternativa de teste de leitura que tem se mostrado efetivo para avaliação de leitura em diversas faixas etárias e níveis de escolarização (Cunha \& Santos, 2010; Mota e cols., 2009; Santos \& Oliveira, 2010; Zucoloto \& Sisto, 2002, Oliveira \& Santos, 2005; Silva \& Santos, 2005). Além disso, é um teste de fácil aplicação e correção,podendo ser usado em diferentes contextos educacionais, inclusive por professores, sem necessitar da intervenção de especialistas.

É um fato constantemente anunciado a necessidade de melhorar o desempenho na leitura de nossos alunos. Diante desse quadro, que requer a prevenção e remediação das dificuldades de leitura, devemos pensar nas implicações práticas dos nossos resultados e daqueles obtidos em outros estudos que usaram o Cloze e em como implementar o uso do instrumento no processo de avaliação de leitura de professores e educadores. Alguns estudos que já estão em andamento buscam normatizar os escores dos testes de Cloze considerando a etapa escolar e as regiões do Brasil (Santos, 2010). Além disso, há pesquisas divulgadas que mostram bons resultados de seu uso enquanto técnica apropriada para programas de intervenção que visam à remediação das atividades de leitura (Santos, 2005; Santos \& Oliveira, 2010).

Diante da complexidade e da necessidade de compreender textos no desenvolvimento de tarefas corriqueiras da vida de um indivíduo, e mais especificamente na vida escolar, este é um passo importante. Não há dúvida de que há uma lacuna no tocante à oferta de instrumentos de avaliação de leitura. Finalmente, a ampla divulgação de resultados favoráveis obtidos com o uso do teste e da técnica favorecerá seu conhecimento e sua utilização por outros pesquisadores e por profissionais que estão no dia a dia com as crianças,os quais poderão incluí-lo em suas práticas pedagógicas.

\section{Referências}

Abraham, R. G., \& Chapelle, C. A. (1992). The meaning of cloze test scores: an item difficulty. The Modern Language Journal, 76, 468479 .

Barnitz, J. G. (1998). Linguistic perspectives in literacy education. The Reading Teacher, 580(2), 110-117.

Bitar, M. L. (1989). Eficiência dos instrumentos de avaliação em leitura. Dissertação de Mestrado, Pontifícia Universidade Católica de São Paulo, São Paulo-SP.

Brandão, A. C. P., \& Spinillo, A. G. (1998). Aspectos gerais e 
específicos na compreensão de textos. Psicologia: Reflexão e Crítica, 11(2), 253-272.

Cantrell, S., Almasi, J., Carter, J., Rintamaa, M., \& Madden, A. (2010). The impact of a strategy-based intervention on the comprehension and strategy use of struggling adolescent readers.Journal of Educational Psychology, 102(2), 257-280.

Chance, L. (1985). Use Cloze encounters of the readability kind for secondary school students. Journal of Reading, 29(8), 690-693.

Cunha, N. B., \& Santos, A. A. A. (2006). Relação entre a compreensão da leitura e a produção escrita em universitários. Psicologia: Reflexão e Crítica, 19, 237-245.

Cunha, N. B., \& Santos, A. A. A. (2010). Estudos de validade entre instrumentos que avaliam habilidades linguísticas. Estudos de Psicologia, 27, 305-314.

Cunha, N. B., \& Santos, A. A. A. (2009). Validade por processo de resposta no teste de Cloze. Fractal: Revista de Psicologia, 21(3), 549-562.

Dias, T. L., Enumo, S. R. F., \& Turini, F. A. (2006). Avaliação do desempenho acadêmico do ensino fundamental de Vitória, Espírito Santo. Estudos de Psicologia-Campinas, 23(4), 381-390.

Ellis, A. W. (1995). Leitura, escrita e dislexia: uma análise cognitiva (D. Batista, Trad., 2a ed.), Porto Alegre: Artes Médicas.

Kintsch, W. (1994). Text comprehension, memory and learning. American Psychologist, 49, 294-303.

Kintsch, W., \& Dijk, T. A. (1978).Toward a model of text comprehension and production. Psychological Review, 85(5), 363-394.

Lucio, P. S., Pinheiro, A. M. V., \& Nascimento, E. (2009). O impacto da mudança no critério de acerto na distribuição dos escores do subteste de leitura no teste de desempenho escolar. Psicologia em Estudo, 14(3), 593-601.

McKenna, M. C., \& Kear, D. J. (1990). Measuring attitude toward reading: A new tool for teachers. The Reading Teacher, 23, 626-639.

Melo, M. T. O. P. (1988). Efeito de um material de história do Brasil para crianças de 8 a 10 anos quanto à inteligibilidade e compreensão do texto. Estudos de Psicologia, 5(2), 112-126.

Milani, R. G. \& Loureiro, S. R. (2009). Crianças em risco psicossocial associado à violência doméstica: o desempenho escolar e o autoconceito como condições de proteção. Estudos de PsicologiaNatal, 14(3), 191-198.

Molina, O. (1979). Avaliação da inteligibilidade de livros didáticos de $1^{\circ}$ e $2^{\circ}$ graus por meio da técnica de Cloze. Tese de Doutorado, Universidade de São Paulo, São Paulo-SP.
Mota, M. M. P. E., Lisboa, R.,Dias, J.,Gontijo, R., Paiva, N.,MansurLisboa, S. F., Silva, D. A., \& Santos, A. A. A.(2009). Relação entre Consciência Morfológica e Leitura Contextual medida pelo teste de Cloze. Psicologia: Reflexão e Crítica, 22(2), 223-229.

Oliveira, K. L., Boruchovitch E., \& Santos, A. A. A. (2008). Leitura e desempenho escolar em português e matemática no ensino fundamental. Paidéia, 18(41), 531-540.

Oliveira, K. L., \& Santos, A. (2008). Estudo de Intervenção para a Compreensão em Leitura na Universidade. Interação em Psicologia, 12(2), 169-177.

Pereira, P. C., Santos, A. B., \& Williams, L. C. A. (2009). Desempenho escolar da criança vitimizada encaminhada ao fórum judicial. Psicologia Teoria e Pesquisa, 25(1), 19-28.

Phakiti, A. (2003). A closer look at the relationship of cognitive and metacognitive strategy use to EFL reading achievement test performance. Language Testing, 20(1), 26-56.

Rego, L., \& Bryant, P. (1993). The connections between phonological, syntactic and semantic skills and children's reading and spelling. European Journal of Psychology, 3, 235-246.

Riley, J. D. (1986). Progressive Cloze as a remedial technique. The Reading Teacher, 39(6), 576-581.

Rupp, A. A., Ferne, T., \& Choi, H. (2006). How assessing reading comprehension with multiple-choice questions shapes the construct: a cognitive processing perspective. Language Testing, 23(4), 441-474.

Sampaio, I. S., \& Santos, A. A. A. (2002). Leitura e Redação entre universitários: avaliação de um programa de remediação. Psicologia em Estudo, 7(1), 31-38.

Santos, A. A. A. (2004). O Cloze como técnica de diagnóstico e remediação da compreensão em leitura. Interação em Psicologia, 8(2), 217-226

Santos, A. A. A. (2005). O Teste de Cloze como instrumento de diagnóstico e de desenvolvimento da compreensão em leitura. Relatório técnico. Itatiba: Universidade São Francisco.

Santos, A. A. A., \& Oliveira, E. Z. (2010). Avaliação e desenvolvimento da compreensão em leitura no ensino fundamental. Psico-USF, $15,93-102$.

Santos, A. A. A., Primi, R., Taxa, F., \& Vendramini, C. M. M. (2002). O teste de Cloze na avaliação da compreensão em leitura. Psicologia: Reflexão e Crítica, 15(3), 549-560.

Silva, M. J. M., \& Santos, A. A. A. (2004). A avaliação da compreensão em leitura e o desempenho acadêmico de universitários. Psicologia em Estudo, 8(3) 459-467. 
Siqueira, A. C., \& DellAglio, D. D. (2010). Crianças e adolescentes institucionalizados: desempenho escolar, satisfação de vida e rede de apoio social. Psicologia Teoria e Pesquisa, 26(3), 407-415.

Spinillo, A. G. (2008). O leitor e o texto: desenvolvendo a compreensão de textos na sala de aula. Interamerican Journal of Psychology, 42, 29-40.

Spinillo, A. G., \& Mahon, E. R. (2007). Compreensão de textos em crianças: comparações entre diferentes classes de inferência a partir de uma metodologia on-line. Psicologia: Reflexão e Crítica, 20(3), 463-471.
Stein, L. M. (1994). TDE - Teste de desempenho escolar: Manual para aplicação e interpretação. São Paulo: Casa do Psicólogo.

Taylor, W. L. (1953). Cloze procedure: A new tool for measuring readability. Journalism Quarterly, 30, 415-433.

Tunmer, W. (1990). The role of language prediction skills in beginning reading. New Zealand Journal of Educational Studies,25(2), 95112.

Zucoloto, K. A., \& Sisto, F. F. (2002). Dificuldades de aprendizagem em escrita e compreensão em leitura. Interação em Psicologia, $6(2), 157-166$

Recebido em: 21/10/2012

Reformulado em: 24/07/2013

Aprovado em: 10/12/2013

\section{Sobre as autoras}

Márcia Maria Peruzzi Elia da Mota (mmotapsi@pesquisador.cnpq.br)

Programa de Pós-Graduação em Psicologia - Universidade Salgado de Oliveira/ Universidade do Estado do Rio de Janeiro

\section{Acácia Aparecida Angeli dos Santos}

Psicóloga; doutora em Psicologia Escolar e do Desenvolvimento Humano pela USP; docente da graduação e da pós-graduação em Psicologia da Universidade São Francisco - Itatiba-SP.

Agradecimentos ao financiamento do CNPq Edital MCT/CNPq 50/2006 - Ciências Humanas, Sociais e Sociais Aplicadas.

Comitê de ética: (protocolo CEP/UFJF 956002 2007) 